José Henrique Hildebrand Grisi Filho

Caracterização de circuitos pecuários com base em redes de movimentação de animais

São Paulo 
José Henrique Hildebrand Grisi Filho

\section{Caracterização de circuitos pecuários com base em redes de movimentação de animais}

Tese apresentada ao Programa de Pós-Graduação em Epidemiologia Experimental Aplicada às Zoonoses da Faculdade de Medicina Veterinária e Zootecnia da Universidade de São Paulo para obtenção do título de Doutor em Ciências

\section{Departamento:}

Medicina Veterinária Preventiva e Saúde Animal

Área de concentração:

Epidemiologia Experimental Aplicada às Zoonoses

Orientador: Prof. Dr. Marcos Amaku

\section{São Paulo}

2012 
Autorizo a reprodução parcial ou total desta obra, para fins acadêmicos, desde que citada a fonte.

\section{DADOS INTERNACIONAIS DE CATALOGAÇÃO-NA-PUBLICAÇÃO}

(Biblioteca Virginie Buff D’Ápice da Faculdade de Medicina Veterinária e Zootecnia da Universidade de São Paulo)

Grisi Filho, José Henrique Hildebrand

Caracterização de circuitos pecuários com base em redes de movimentação de animais / José Henrique Hildebrand Grisi Filho. -- 2012.

$30 \mathrm{f.}$ : il.

Tese (Doutorado) - Universidade de São Paulo. Faculdade de Medicina Veterinária e Zootecnia. Departamento de Medicina Veterinária Preventiva e Saúde Animal, São Paulo, 2012.

Programa de Pós-Graduação: Epidemiologia Experimental e Aplicada às Zoonoses.

Área de concentração: Epidemiologia Experimental e Aplicada às Zoonoses.

Orientador: Prof. Dr. Marcos Amaku.

1. Epidemiologia. 2. Análise de redes. 3. Análise de comunidades. 4. Circuito pecuário. 5. Movimentação animal. I. Título. 


\section{CERTIFICADO}

Certificamos que o Projeto intitulado "Caracterização de circuitos pecuários com base em redes de movimentação de animais", não utilizando animais, protocolado sob o n $1619 / 2009$, sob a responsabilidade do Prof. Dr. Marcos Amaku, está de acordo com os princípios éticos de experimentação animal da Comissão de Bioética da Faculdade de Medicina Veterinária e Zootecnia da Universidade de São Paulo e foi aprovado em reunião de 22/04/09.

We certify that the Research "Characterization of zones based on animal movements networks", protocol number 1619/2009, under the responsibility Prof. Dr. Marcus Amaku, agree with Ethical Principles in Animal Research adopted by Bioethic Commission of the School of Veterinary Medicine and Animal Science of University of São Paulo and was approved in the meeting of day $04 / 22 / 09$.

São Paulo, 23 de abril de 2009

Profa Dra Denise Tabacchi Fantoni

Vice-Presidente da Comissão de Bioética FMVZ/USP 


\section{FOLHA DE AVALIAÇÃO}

Nome: Grisi Filho, José Henrique Hildebrand

Título: Caracterização de circuitos pecuários com base em redes de movimentação de animais

Tese apresentada ao Programa de Pós-Graduação em Epidemiologia Experimental Aplicada às Zoonoses da Faculdade de Medicina Veterinária e Zootecnia da Universidade de São Paulo para obtenção do título de Doutor em Ciências

Data:

BANCA EXAMINADORA

Prof. Dr. :

Instituição: Julgamento:

Prof. Dr. :

Instituição: Julgamento:

Prof. Dr. :

Instituição: Julgamento:

Prof. Dr. :

Instituição: Julgamento:

Prof. Dr. :

Instituição: Julgamento: 


\section{Agradecimentos}

Projeto financiado pela FAPESP e CNPq.

Ao Prof. Dr. Marcos Amaku, pela orientação, sabedoria, paciência e confiança;

Ao Prof. Dr. Fernando Ferreira, por toda a ajuda, sugestões, apoio e amizade;

Aos colegas Raul e Rísia, pelo trabalho em equipe sem o qual esta tese não seria a mesma;

À todos os colegas do LEB que fazem deste um excelente local de trabalho;

À minha família, por tudo;

À todos que contribuíram, de forma direta ou indireta, para a conclusão desta tese. 


\section{RESUMO}

GRISI-FILHO, J. H. H. Caracterização de circuitos pecuários com base em redes de movimentação de animais. [Characterization of production zones based on animal movement networks.] 2012. 30 f. Tese (Doutorado em Ciências) - Faculdade de Medicina Veterinária e Zootecnia, Universidade de São Paulo, São Paulo, 2012.

Uma rede é um conjunto de nós conectados entre si através de um conjunto de arestas. Redes podem representar qualquer conjunto de objetos que possuam relações entre si. Comunidades são conjuntos de nós relacionados de uma maneira significativa, provavelmente compartilhando propriedades e/ou atuando de forma similar dentro de uma rede. Quando a análise de redes é aplicada ao estudo de padrões de movimentação animal, as unidades epidemiológicas de interesse (propriedades, estabelecimentos, municípios, estados, países, etc) são representadas como nós, enquanto a movimentação animal entre elas é representada através das arestas de uma rede. Descobrir a estrutura de uma rede, e portanto as preferências e rotas comerciais, pode ser útil para um pesquisador ou gestor de saúde animal. Foi implementado um algoritmo de detecção de comunidades para encontrar grupos de propriedades que é consistente com a definição de circuito pecuário, assumindo que uma comunidade é um grupo de nós (fazendas, abatedouros) no qual um animal vai mais provavelmente permanecer durante sua vida. Este algoritmo foi aplicado na rede interna de movimentação animal de 2007 do Estado do Mato Grosso. Esse banco de dados contém informação sobre 87.899 propriedades e 521.431 movimentações durante o ano, totalizando 15.844 .779 de animais movimentados. O algoritmo de detecção de comunidades encontrou uma partição da rede que mostra um claro padrão geográfico e comercial, duas importantes características para aplicações em medicina veterinária preventiva, além de possuir uma interpretação clara e significativa em redes de comércio onde ligações se estabelecem a partir da escolha dos nós envolvidos.

Palavras-chave: Epidemiologia. Análise de redes. Análise de comunidades. Circuito pecuário. Movimentação animal. 


\section{ABSTRACT}

GRISI-FILHO, J. H. H. Characterization of production zones based on animal movement networks. [Caracterização de circuitos pecuários com base em redes de movimentação de animais.] 2012. 30 f. Tese (Doutorado em Ciências) - Faculdade de Medicina Veterinária e Zootecnia, Universidade de São Paulo, São Paulo, 2012.

A network is a set of nodes that are linked together by a set of edges. Networks can represent any set of objects that have relations among themselves. Communities are sets of nodes that are related in an important way, probably sharing common properties and/or playing similar roles within a network. When network analysis is applied to study the livestock movement patterns, the epidemiological units of interest (farm premises, counties, states, countries, etc.) are represented as nodes, and animal movements between the nodes are represented as the edges of a network. Unraveling a network structure, and hence the trade preferences and pathways, could be very useful to a researcher or a decision-maker. We implemented a community detection algorithm to find livestock communities that is consistent with the definition of a livestock production zone, assuming that a community is a group of farm premises in which an animal is more likely to stay during its life time than expected by chance. We applied this algorithm to the network of within animal movements made inside the State of Mato Grosso, for the year of 2007. This database holds information about 87,899 premises and 521,431 movements throughout the year, totalizing $15,844,779$ animals moved. The community detection algorithm achieved a network partition that shows a clear geographical and commercial pattern, two crucial features to preventive veterinary medicine applications, and also has a meaningful interpretation in trade networks where links emerge from the choice of trader nodes.

Keywords: Epidemiology. Network analysis. Community analysis. Livestock production zone. Animal movement. 
SUMÁRIO

1 INTRODUÇÃO $\quad 9$

1.1 Redes . . . . . . . . . . . . . . . . . . . . . 9

1.2 Análise de Comunidades em Medicina Veterinária . . . . . . . . . . 10

1.3 Definição de Comunidade e Circuitos Pecuários . . . . . . . . . . . . 11

2 MATERIAIS E MÉTODOS 14

2.1 Base de dados . . . . . . . . . . . . . . . . . . . . . . . . . . 14

2.2 Detecção de circuitos pecuários . . . . . . . . . . . . . . . 14

2.2 .1 Otimização da função de modularidade . . . . . . . . . . . . . 16

2.2 .2 Obtenção de uma solução única . . . . . . . . . . . . . . . . . . . . 18

2.3 Avaliação da performance do algoritmo f . . . . . . . . . . . . 19

2.3 .1 Escalonamento multidimensional $\ldots \ldots \ldots \ldots$

2.4 Softwares utilizados $\ldots \ldots \ldots \ldots \ldots$

3 RESULTADOS 21

4 DISCUSSÃO 26

5 CONCLUSÕES $\quad 27$

$\begin{array}{ll}\text { REFERÊNCIAS } & 28\end{array}$ 


\section{INTRODUÇÃO}

\section{$1.1 \quad$ Redes}

Uma rede é um conjunto de nós (ou vértices) conectados entre si através de um conjunto de arestas (ou ligações), como demonstrado na figura 1.1. Redes podem representar qualquer conjunto de objetos que possuam relações entre si. Podem, por exemplo, representar as relações sociais entre indivíduos (figura 1.2). Em uma rede ponderada cada aresta possui um valor, que representa a intensidade da ligação entre dois nós. Redes podem ser direcionadas e não-direcionadas: uma rede direcionada apresenta a direção da relação entre dois nós; uma rede não-direcionada assume que a relação entre dois nós é sempre recíproca. Em uma rede direcionada e ponderada, o grau de entrada é a soma dos valores das arestas que um nó recebe, o grau de saída é a soma dos valores das arestas que um nó envia e o grau total é igual à soma dos graus de entrada e de saída.

Figura 1.1 - Ilustração de uma rede, com indicação de nós e vértices

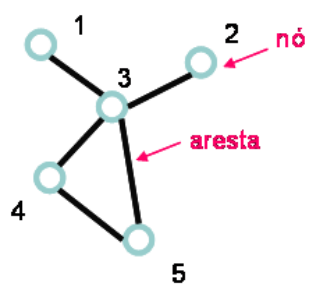

Figura 1.2 - Rede de associações frequentes entre 62 golfinhos de Doubtful Sound, New Zealand (LUSSEAU et al., 2003)

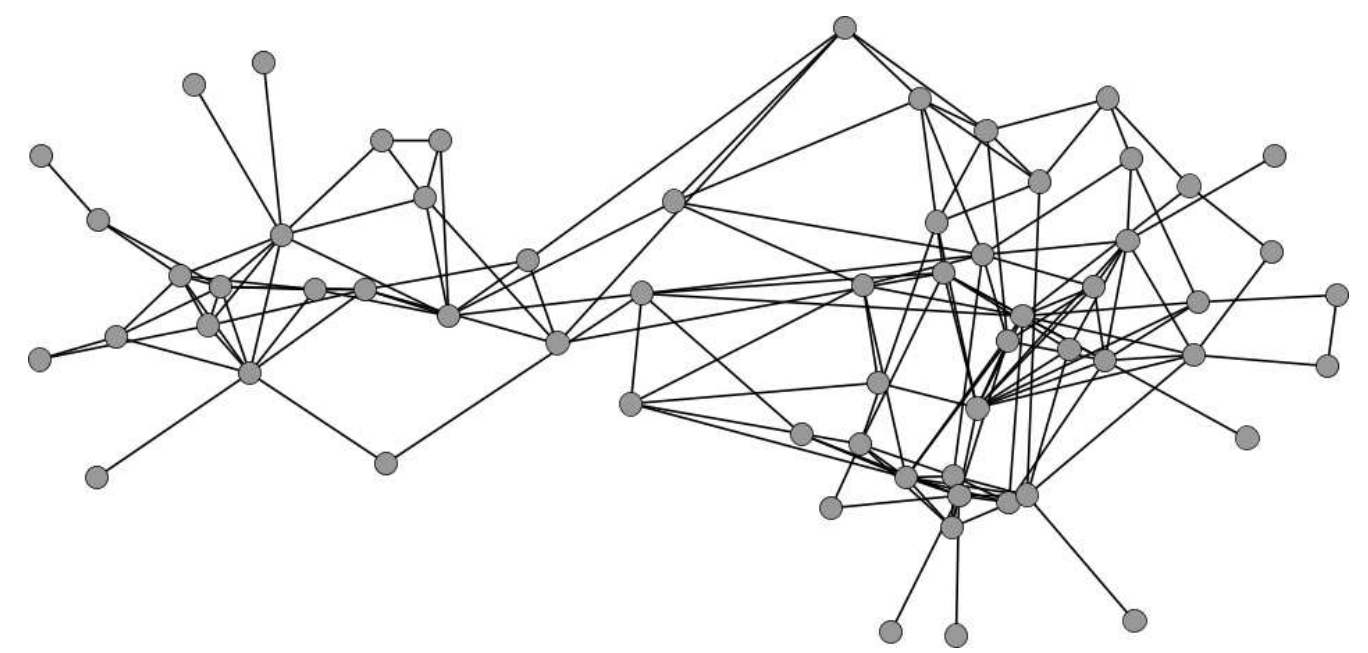


Quando a análise de redes é aplicada ao estudo da movimentação animal dentro de um sistema produtivo, as unidades epidemiológicas de interesse (propriedades, estabelecimentos, municípios, estados, países, etc) são representadas como nós, enquanto a movimentação animal entre elas é representada através das arestas de uma rede, onde o valor de cada aresta traz o número de animais comercializados entre dois nós. Assim, o grau de entrada de um nó equivale à quantidade de animais comprados e o grau de saída equivale à quantidade de animais vendidos por uma propriedade. A análise de redes têm sido utilizada para estudar uma grande variedade de problemas dentro da medicina veterinária, incluindo análises de epidemias (GIBBENS, WILESMITH e SHARPE, 2001), análises etológicas (LUSSEAU e NEWMAN, 2004), padrões de distribuição de doenças, modelos preditivos (HARVEY et al., 2007), análises de risco, eficácia de sistemas de vigilância entre outros. MARTÍNEZ-LOPEZ, PEREZ e SANCHEZ-VIZCAÍNO (2009) fazem uma revisão sobre o assunto.

\subsection{Análise de Comunidades em Medicina Veterinária}

Comunidades são conjuntos de nós relacionados entre si de uma maneira significativa, provavelmente compartilhando propriedades e/ou atuando de forma similar dentro de uma rede. Estudos recentes utilizaram a análise de comunidades em redes de trânsito animal com o intuito de revelar a estrutura subjacente da rede (GREEN, GREGORY e MUNRO, 2009) (GREEN et al., 2011) (LENTZ et al., 2011).

Descobrir essa estrutura e portanto as preferências de comércio entre propriedades pode se mostrar útil para um pesquisador ou tomador de decisão. Em primeiro lugar, permite a identificação dos fluxos de comércio entre zonas de produção, possibilitando um melhor entendimento do sistema de produção animal. A existência e funcionalidade de grupos dentro de uma rede pode influenciar o espalhamento de doenças, alterando a velocidade de espalhamento, o tamanho final e a duração de uma epidemia (SALATHÉ e JONES, 2010). Análises de comunidade poderiam ser usadas para determinar os grupos de uma amostra estratificada que visa levar em consideração o trânsito animal, ou ajudar no planejamento de um sistema de vigilância voltado ao risco, escolhendo grupos de nós 
sob risco que possuam intenso comércio entre si. Seria possível, na ocasião de erradicação de uma doença específica, dividir um programa sanitário em etapas nas quais um grupo de municípios é sanitizado por vez, caso esses municípios apresentem baixo risco de reinfecção através de movimentos oriundos de fora do grupo. Propriedades que compartilham preferências comerciais podem compartilhar práticas de manejo e biossegurança, e serem selecionadas para um programa sanitário direcionado. A Organização Mundial de Saúde Animal (OIE), define em seu Terrestrial Animal Health Code (WORLD ORGANISATION FOR ANIMAL HEALTH, 2010), os conceitos de zoneamento, regionalização e compartimento. Dada a dificuldade de estabelecer e manter um status livre de doença para o território de um país inteiro, pode ser vantajoso estabelecer e manter uma subpopulação com status sanitário diferenciado dentro de seu território. Assim, a delimitação de zonas sanitárias dentro de uma região pode levar em consideração não apenas municípios com as mesmas condições sanitárias, mas também regiões que possuam intenso comércio com estes. A análise de comunidades pode ajudar no planejamento da fronteira deste tipo de área, caso haja isolamento de uma subpopulação (p.ex, com restrição de movimentação), bloqueando o comércio entre fazendas de áreas diferentes: essa abordagem poderia otimizar a divisão de tal região e avaliar quanta perda econômica é esperada devido a restrição de comércio após uma zona específica ser estabelecida.

Esses são apenas alguns exemplos. Como uma técnica exploratória, suas aplicações são virtualmente infinitas, provendo suporte na formulação de novas hipóteses, na avaliação de premissas, na seleção de futuras ferramentas analíticas e no planejamento de futuras coletas de dados.

\subsection{Definição de Comunidade e Circuitos Pecuários}

A tarefa de dividir uma rede em comunidades, intuitiva a princípio, não é realmente bem definida. Os principais elementos do problema, i.e. os conceitos de comunidade e cluster, não estão rigorosamente definidos, requerendo um grau de arbitrariedade e/ou bom senso. Uma grande variedade de algoritmos e ferramentas foram desenvolvidos para revelar a estrutura comunitária de uma rede (FORTUNATO, 2010), e a escolha de qual 
algoritmo utilizar deve levar em consideração as definições e premissas do modelo, e se os mesmos são adequados para resolver o problema em questão.

Como notado por BIGRAS-POULIN et al. (2006), em redes de movimentação de gados de corte as fazendas agem no geral como geradoras (algumas com grau de entrada igual a zero, chamadas de "fazendas de cria") de gado, e abatedouros agem como escoadouros. Esses dois tipos de comportamento impõem um fluxo direcionado à rede. É portanto razoável assumir que a direção dos movimentos não pode ser ignorada sem a perda de informação importante, e é melhor usar ferramentas que levem em consideração essa direção sempre que possível.

As poucas aplicações recentes de detecção de comunidades em redes de trânsito animal que consideram a direção dos movimentos (GREEN, GREGORY e MUNRO, 2009) (GREEN et al., 2011) usam a definição de comunidade dada por NEWMAN e GIRVAN (2004) e adaptada a redes direcionadas por LEICHT e NEWMAN (2008): um conjunto de nós é uma comunidade se o número de ligações internas a este exceder o esperado para um mesmo conjunto de nós no modelo nulo (FORTUNATO, 2010). O que equivale a dizer que os nós que formam uma comunidade são mais densamente conectados entre si do que um conjunto similar que não forma uma comunidade. Essa definição é bastante útil e intuitiva, mas não é a única definição de comunidade possível.

KIM, SON e JEONG (2010) desenvolveram um método para detecção de comunidades que leva em consideração a direcionalidade e o peso das ligações, e que possui melhor performance que o método desenvolvido por LEICHT e NEWMAN (2008) em alguns casos, trazendo uma definição de comunidade mais adequada em algumas situações. Esse método é baseado no cálculo do LinkRank, um conceito derivado do PageRank do Google (PAGE et al., 1998). Nessa definição, uma comunidade é definida como um grupo de nós nos quais um passeador aleatório permanece mais provavelmente do que o esperado pelo acaso. Se definirmos um circuito pecuário como o conjunto de localidades pelas quais um animal passará durante o ciclo produtivo, então essa definição de comunidade é mais adequada para encontrar tais grupos, especialmente se desejamos compreender os fluxos 
entre zonas de produção de uma região ou país. Uma comunidade seria então definida como um grupo de locais onde um animal permanecerá mais provavelmente durante sua vida (o ciclo produtivo) do que o esperado pelo acaso.

Assim, o objetivo deste estudo foi implementar e testar um método de detecção de comunidades que identifique circuitos pecuários em uma rede de movimentação de animais. 


\section{MATERIAIS E MÉTODOS}

\subsection{Base de dados}

Foi analisada a rede interna de movimentação de bovinos do Estado do Mato Grosso do ano de 2007 (movimentos realizados entre 01/01/2007 e 31/12/2007), fornecida pelo INDEA (Instituto de Defesa Agropecuária do Estado do Mato Grosso). Essa base de dados contém informações sobre 87.899 propriedades (fazendas, frigoríficos, eventos, etc) e 521.431 movimentos ao longo do ano, totalizando 15.844 .779 de animais movimentados. O Estado do Mato Grosso é o terceio maior do Brasil, com uma àrea de $903.357 \mathrm{~km}^{2}$ e o maior rebanho nacional, com aproximadamente 28.757 .438 bovinos (13\% do rebanho nacional em 2010), sendo em sua maioria rebanhos de exploração de corte (INSTITUTO BRASILEIRO DE GEOGRAFIA E ESTATÍSTICA, 2010). Está localizado na região centro oeste do país e possui 141 municípios.

Foram extraídas informações sobre as propriedades de origem e destino e sobre o número de animais movimentados. Essas informações foram então resumidas para a menor divisão pública administrativa, agrupando os movimentos feitos por propriedades que pertenciam ao mesmo município. A rede analisada possui portanto 141 nós (municípios) e 3.980 arestas, estas últimas ponderadas pelo total de animais movimentados entre dois municípios durante o ano de 2007. A figura 2.1 traz uma representação dessa rede, onde cada nó está localizado no centróide no respectivo município (aproximadamente).

\subsection{Detecção de circuitos pecuários}

Uma partição $C$ é uma divisão de um conjunto de $N$ objetos em clusters (ou grupos) não vazios e não sobrepostos. Os termos clusters, grupos e comunidades serão utilizados como sinônimos no presente trabalho. É possível representar uma partição $C$ como um vetor de tamanho $N$, no qual o i-ésimo elemento de $C$ é igual o grupo ao qual pertence o objeto $i$. Assim, uma partição $C=\{\{1\},\{1\},\{2\},\{2\}\}$ é a divisão de um conjunto de 4 objetos em dois grupos, nos quais os dois primeiros objetos foram designados ao primeiro grupo e os dois últimos objetos foram designados ao segundo 
Figura 2.1 - Rede de movimentação interna de animais do Mato Grosso durante o ano de 2007. Movimentos agrupados por munícipios

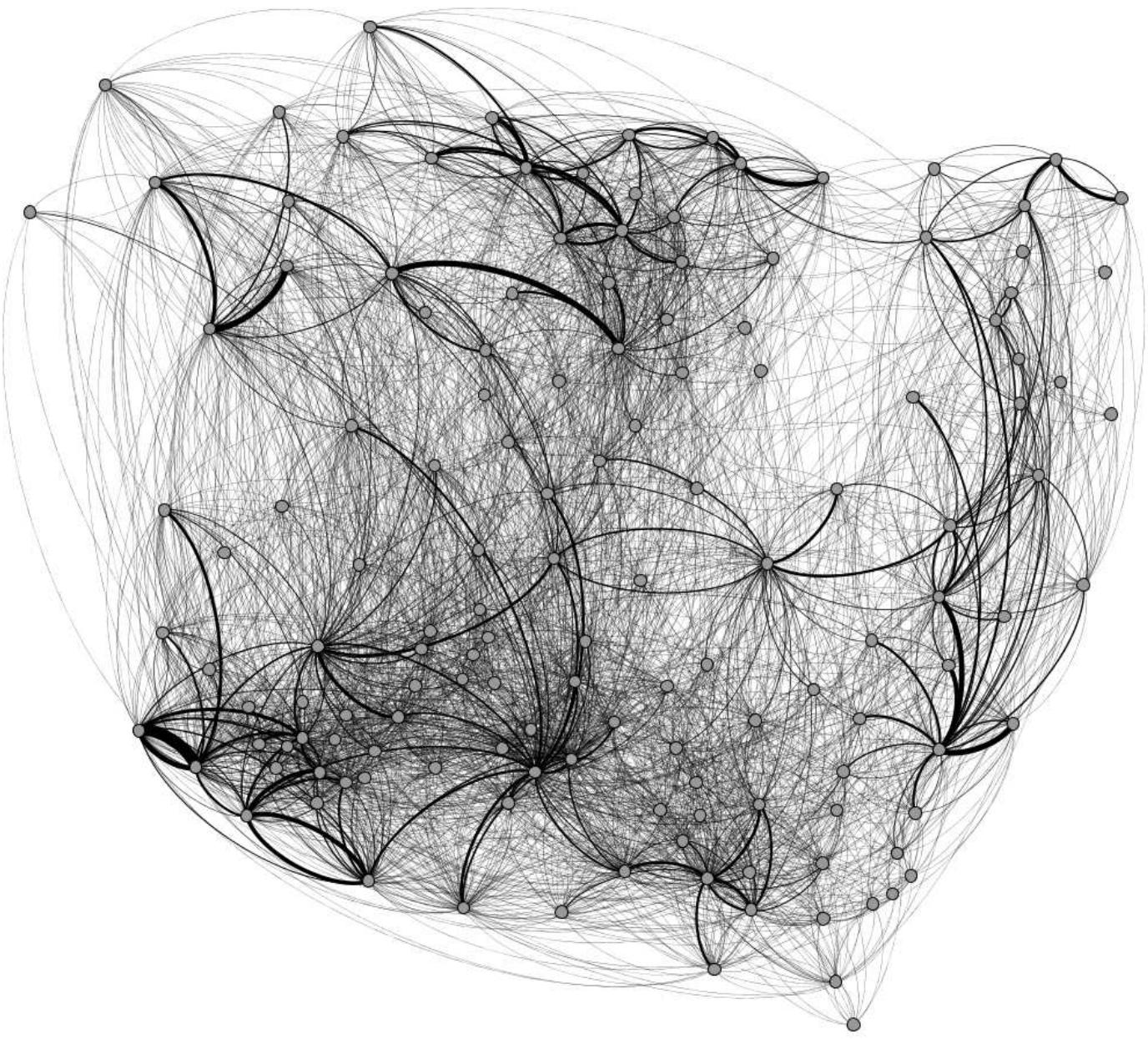

grupo. Da definição de comunidades emerge uma função chamada modularidade, a qual descreve a qualidade de qualquer partição em descrever a estrutura modular presente em uma rede. Quanto maior o valor de modularidade de uma partição, melhor essa partição reflete a estrutura da rede. A função de modularidade proposta por KIM, SON e JEONG (2010) pode ser calculada usando a seguinte equação:

$$
Q^{l r}=\sum_{i j}\left[L_{i j}-\pi_{i} \pi_{j}\right] \delta_{c_{i} c_{j}}
$$

onde $Q^{l r}$ é a modularidade de uma dada partição; $L_{i j}$ é o valor de LinkRank da aresta que conecta os nós $i$ e $j ; \pi_{i}$ é o valor de PageRank do nó $i ; \pi_{j}$ é o valor de PageRank do 
nó $j$; e $\delta_{c_{i} c_{j}}=1$ caso os nós $i$ e $j$ sejam designados à mesma comunidade e $\delta_{c_{i} c_{j}}=0$ caso contrário. O valor do LinkRank de uma dada aresta entre os nós $i$ e $j$ é uma medida de sua importância, dada por:

$$
L_{i j}=\pi_{i} G_{i j}
$$

onde $\pi_{i}$ é o valor de PageRank (PAGE et al., 1998) do nó $i$ e $G$ é a Matriz Google ("Google Matrix", no original), uma matriz de probabilidade para o processo de passeio aleatório. O PageRank é um algoritmo de análise de ligações que produz um rank global da importância de todos os nós de uma rede, baseado na matriz de probabilidade do processo de passeio aleatório (também chamado de "caminhada do bêbado"). O cálculo do PageRank leva em consideração o grau de entrada de um dado nó e o grau de entrada de seus vizinhos. É uma medida propagada através da rede, baseada na premissa de que nós importantes recebem ligações de nós importantes (figura 2.2). Para maiores detalhes, veja LANGVILLE e MEYER (2006) e KIM, SON e JEONG (2010).

Figura 2.2 - PageRank calculado em rede simples

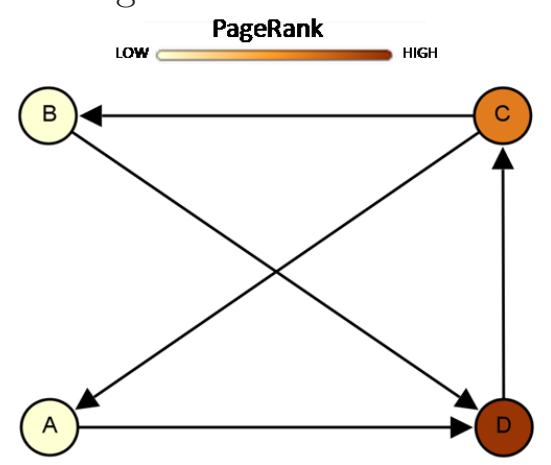

Figura 2.2 - Legenda:

Os nós $\mathrm{A}, \mathrm{B}$ e $\mathrm{C}$ recebem o mesmo número de ligações (i.e. grau de entrada igual a 1). No entanto, o nó C possui um maior valor de PageRank que A e B, porque a ligação que C recebe provém de um nó importante (com maior grau de entrada, nó D), elevando assim seu PageRank.

\subsubsection{Otimização da função de modularidade}

Para otimizar a função de modularidade e encontrar uma partição ótima da rede, foi utilizado o algoritmo de Arrefecimento Simulado (Simulated Annealing - SA) (KIRKPATRICK, GELATT e VECCHI, 1983). Esse algoritmo é um método de otimização baseado em um procedimento probabilístico que evita que a solução encontrada pertença 
a um máximo local, e já foi aplicado para a otimização de modularidade anteriormente por GUIMERÀ e AMARAL (2005), por KIM, SON e JEONG (2010) e por GOOD, DE MONTJOYE e CLAUSET (2010).

No estado inicial, cada nó é designado à sua própria comunidade. Em cada passo do algoritmo, um nó aleatório é designado à uma comunidade aleatória, e a mudança $\Delta Q^{l r}$ na modularidade é computada:

$$
\Delta Q^{l r}=Q_{n}^{l r}-Q_{o}^{l r}
$$

onde $Q_{n}^{l r}$ é a modularidade da nova partição e $Q_{o}^{l r}$ é a modularidade da partição anterior. Se $\Delta Q^{l r}>0$, a mudança é aceita e a nova partição é assimilada para o próximo passo. Se $\Delta Q^{l r} \leq 0$ então há uma probabilidade $P\left(\Delta Q^{l r}\right)$ de que a mudança será aceita:

$$
P\left(\Delta Q^{l r}\right)=\exp \left(\frac{\Delta Q^{l r}}{T}\right)
$$

onde $T$ é um número real positivo, representando a temperatura atual do sistema. Talvez valha a pena mencionar que $T$ não se trata de uma temperatura real. $O$ método de arrefecimento simulado foi inspirado na termomecânica, e a repetição desse processo durante tempo suficiente simula o movimento de átomos de um material sob processo de recozimento à uma temperatura $T$, utilizado para obtenção de estados de baixa energia de um sólido, daí o termo.

Depois de $I$ iterações o sistema é "resfriado", multiplicando a temperatura presente $T$ por um fator de resfriamento $c(0<c<1)$. Este processo se repete até que o sistema esteja "congelado", ou seja, nenhuma mudança proposta é aceita durante o ciclo de uma temperatura. Isso significa que em altas temperaturas o sistema irá explorar muitas configurações, e à medida em que o algoritmo avança e a temperatura decresce, o sistema passa a se concentrar nas melhores soluções, evitando mudanças que decresçam muito o valor de modularidade. O número limite de iterações $I$, o valor da temperatura incial $T_{i}$ e o fator de resfriamento $c$ devem ser ajustados para cada função específica a ser otimizada. 
Neste trabalho foram inicialmente utilizados os valores sugeridos por GUIMERÀ e AMARAL (2005), posteriormente ajustados até a obtenção de um desempenho satisfatório, de maneira que o algoritmo teste um conjunto suficientemente grande de soluções. Os valores foram ajustados em $I=N^{2}$ ( $N=$ número de nós da rede), $T_{i}=1.000$ e $c=0,95$.

Para reduzir o tempo gasto pelo algoritmo, foi gerada uma matriz de Modularidade $M$, de tamanho $N$ x $N$ onde $M_{i j}$ é o resultado de $L_{i j}-\pi_{i} \pi_{j}$, o termo dentro dos colchetes da equação 2.1. Assim, essa matriz $M$ contém a contribuição à modularidade total caso os nós $i$ e $j$ sejam designados à mesma comunidade, possibilitando o cálculo direto de $\Delta Q^{l r}$, evitando o cálculo da modularidade total da partição testada a cada passo.

\subsubsection{Obtenção de uma solução única}

GOOD, DE MONTJOYE e CLAUSET (2010) mostraram que redes com estrutura modular apresentam frequentemente um alto número de degenerações, partições de alta modularidade que escondem a partição situada no máximo global (melhor solução). Assim, frequentemente redes exibirão não apenas um claro máximo global de modularidade, mas um platô de soluções, todas com alta modularidade, não muito diferentes entre si. Embora isso pareça impor um obstáculo para a detecção de comunidades dentro de uma rede, na verdade mostra a possibilidade de escolher uma divisão da rede que atenda necessidades específicas de uma situação sem violar a definição de comunidade previamente definida. Ao invés de analisar todas as partições de alta modularidade, pode ser útil extrair padrões comuns dessas partições, revelando onde elas concordam, i.e. quais nós aparecem juntos em todas as soluções encontradas. A solução final pode ser encarada como "blocos de construção" ("building blocks"), os quais podem ser submetidos posteriormente a um método de aglomeração adequado.

Para resumir a informação obtida, foram selecionados os melhores resultados (patições com valores de modularidade até 1\% abaixo do máximo encontrado) e identificados como comunidades os grupos de municípios que foram designados para o mesmo cluster em todas as partições selecionadas. Isso foi possível através da multiplicação el- 
emento a elemento (produto Hadamard) das matrizes de similaridade de cada partição selecionada:

$$
S m=S m_{1} \circ S m_{2} \circ \ldots \circ S m_{n}
$$

onde $S m$ é a matriz de similaridade correspondente à partição final $C$ e $S m_{n}$ é a matriz de similaridade correspondente à partição $C_{n}$. Uma matriz de similaridade é uma matriz esparsa de tamanho $N \times N$, onde o elemento $S m_{i j}=1$ se os nós $i$ e $j$ pertencem ao mesmo cluster e 0 caso contrário. Tendo como estrutura comunitária a partição $C$, foi possível analisar o comércio de animais intra e intercomunitário.

\subsection{Avaliação da performance do algoritmo}

Uma maneira útil de avaliar se as partições obtidas são similares ou diferentes entre si é a distância de Variação de Informação (Variation of Information - VI) (MEILA, 2007). A distância VI é uma métrica baseada em teoria da informação e entropia, e mede a quantidade de informação não compartilhada entre duas partições. A entropia associada a uma partição $C$ é dada pela seguinte equação:

$$
H(C)=-\sum_{k=1}^{k} P(k) \log P(k)
$$

onde $P(k)$ é a probabilidade de escolher um objeto aleatório da partição $C$ que pertença à comunidade $k$. Loco $H(C)$ provê a incerteza total a respeito de $C$. Define-se $P\left(k, k^{\prime}\right)$ como a probabilidade de um objeto pertencer à comunidade $k$ na partição $C$ e simultaneamente à comunidade $k^{\prime}$ na partição $C^{\prime}$, ou seja, a distribuição conjunta das variáveis aleatórias associadas às duas partições. Então a informação mútua entre as partições $C$ e $C^{\prime}$ é dada por:

$$
I\left(C, C^{\prime}\right)=\sum_{k=1}^{K} \sum_{k^{\prime}=1}^{K^{\prime}} P\left(k, k^{\prime}\right) \log \frac{P\left(k, k^{\prime}\right)}{P(k) P^{\prime}\left(k^{\prime}\right)}
$$

e essa é a quantidade de incerteza sobre $C^{\prime}$ que é reduzida se conhecermos $C$. A Variação de Informação pode então ser definida da seguinte maneira:

$$
V I\left(C, C^{\prime}\right)=H(C)+H\left(C^{\prime}\right)-2 I\left(C, C^{\prime}\right)
$$


Quando aplicada a duas partiões iguais $V I=0$. Quando aplicada a partições extremas que não compartilham nenhuma informação, como por exemplo a partição onde cada nó pertence à sua própria comunidade $C=\{\{1\},\{2\}, \ldots,\{N\}\}$ e a partição onde todos os nós pertencem à mesma comunidade $C^{\prime}=\{k\}$, o valor de VI é o máximo possível, $V I=\log N$ (onde $N$ é o tamanho das partições analisadas). Essa distância foi calculada entre a solução final, todas as soluções dadas pelo algoritmo SA e 50 partições aleatórias. Esse conjunto de distâncias pode ser organizado em uma matriz de distância simétrica $D$, onde $D_{i j}=D_{j i}$, que representa a VI entre as partições $C_{i}$ e $C_{j}$.

\subsubsection{Escalonamento multidimensional}

Para facilitar a interpretação da magnitude das distâncias encontradas, optou-se por projetar a matriz $D$ em um plano euclidiano utilizando a técnica de escalonamento multidimensional. O escalonamento multidimensional (Multidimensional Scaling - MDS) constrói uma configuração de $n$ pontos em um espaço euclidiano tal que as distâncias entre pontos coincida, tanto quanto possível, as dissimilaridades originais entre $n$ objetos (COX e COX, 2000).

\subsection{Softwares utilizados}

A função de modularidade, o algoritmo SA e o código para obtenção de uma solução única foram implementados no software R (R DEVELOPMENT CORE TEAM, 2010). Os mapas foram produzidos no software R (R DEVELOPMENT CORE TEAM, 2010), utilizando os seguintes pacotes: "PBSmapping" (SCHNUTE et al., 2010), "maptools" (LEWIN-KOH et al., 2011), "sp” (BIVAND, PEBESMA e GOMEZ-RUBIO, 2008) e "foreign" (R DEVELOPMENT CORE TEAM, 2011). As representações gráficas de redes foram produzidas no software Gephi (BASTIAN, HEYMANN e JACOMY, 2009). As distâncias VI foram calculadas utilizando o pacote "mcclust" (FRITSCH, 2009). 


\section{RESULTADOS}

Após 50 iterações, o algoritmo SA foi capaz de identificar 24 partições únicas que maximizam a função de modularidade. Essas partições são muito similares entre si, tanto em termos de informação como em valores de modularidade (figura 3.1).

Figura 3.1 - Partições amostradas e superfície de modularidade.

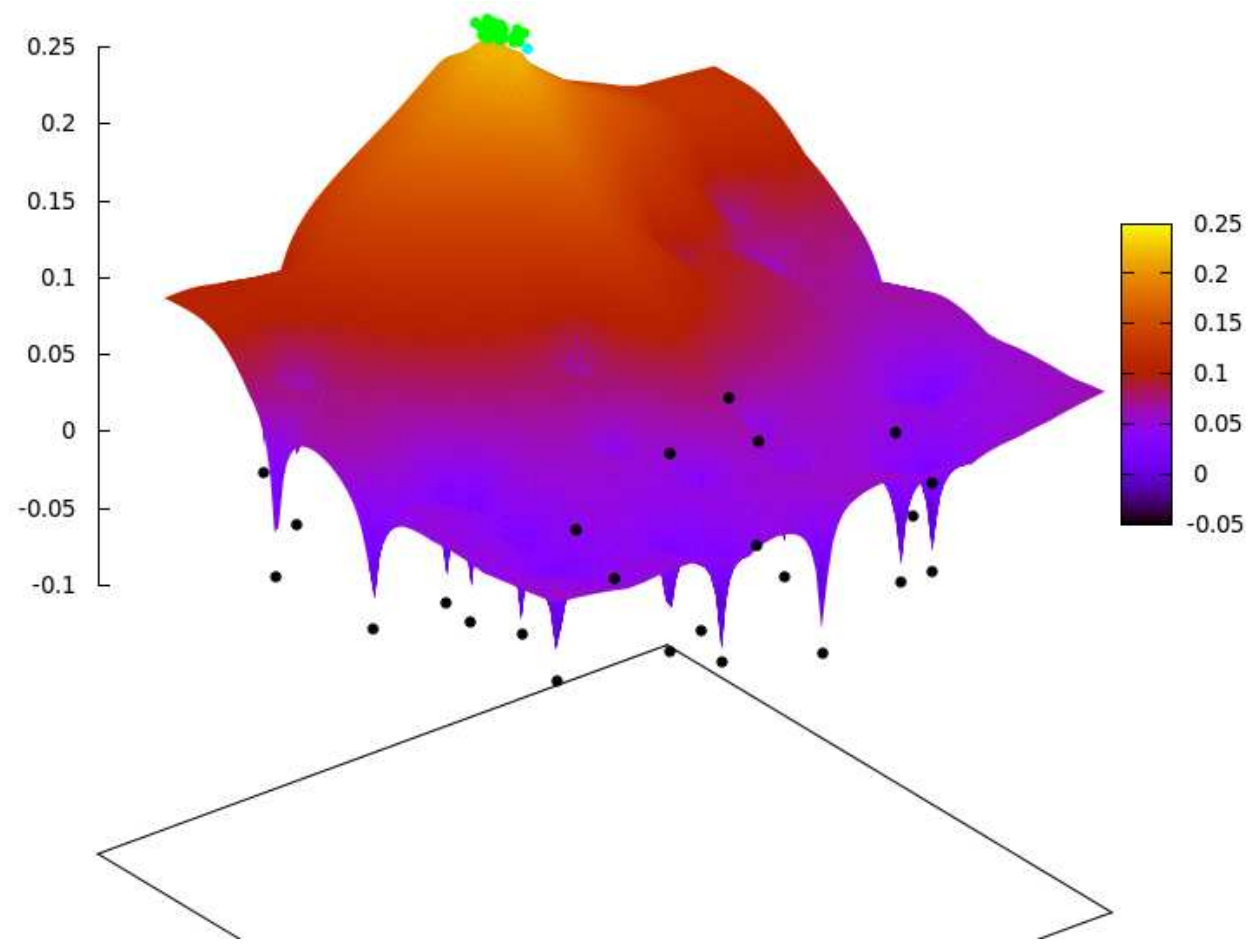

Figura 3.1 - Legenda:

Distância entre os pontos nos eixos $x$ e $y$ representam a distância VI (stress $=23.89$ ). Pontos verdes são partições obtidas através do algoritmo SA, pontos pretos são partições aleatórias e o pronto azul claro é a partição final. A superfície colorida é a função de modularidade, reconstruída a partir de todas as partições analisadas. Partições obtidas através do algoritmo SA estão localizadas no máximo da função de modularidade, muito próximas umas das outras quando comparadas com as partições aleatórias. Ainda, a partição final permanece perto das soluções do SA, e sua flexibilidade é obtida com o sacrifício de uma pequena parcela de sua modularidade.

A solução final, que resume a informação de todas as outras partições, retém um bom valor de modularidade, apresentando uma alta flexibilidade para a definição de clusters sem violar a definição de comunidade escolhida. Esta possui 11 comunidades, sendo que 2 municípios permaneceram desagrupados, dado que eles foram designados a comunidades distintas por duas ou mais partições (figuras 3.2 e 3.3). Apenas três 
municípios foram designados a comunidades não adjacentes, mostrando que a solução em questão possui um claro padrão geográfico.

Figura 3.2 - Circuitos pecuários do Mato Grosso (detalhado)

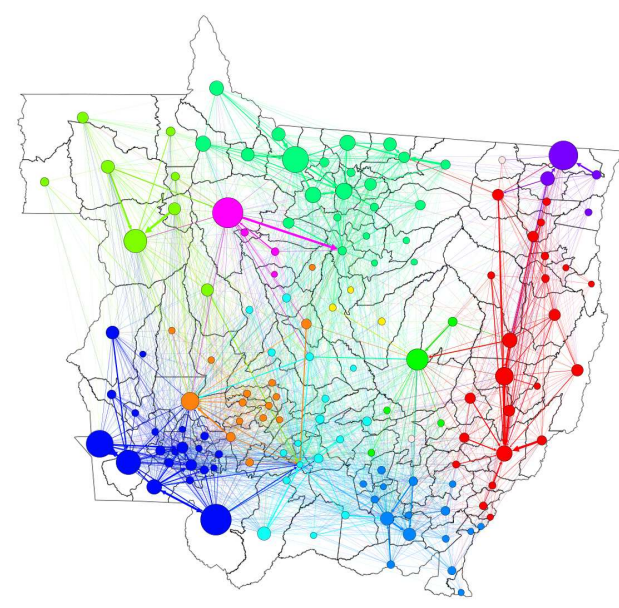

(a) Movimentos internos

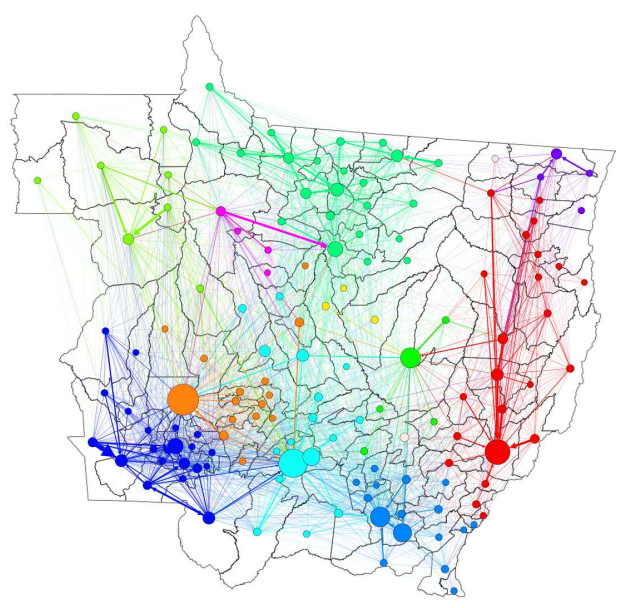

(b) PageRank

Figura 3.2 - Legenda:

A posição dos nós é dada pelo centróide do município correspondente (aproximado). As cores dos nós identificam as 11 comunidades encontradas. Nós brancos permaneceram desagrupados. O tamanho da aresta é proporcional ao número de animais movimentados entre dois municípios, e sua cor identifica a comunidade de origem de uma ligação. Na figura 3.2(a) o tamanho dos nós é proporcional ao número de animais comercializados internamente. Na figura 3.2(b) o tamanho dos nós é proporcional ao valor de PageRank.

A partição encontrada mostra também um claro padrão comercial. Comunidades detectadas preferem comercializar animais internamente (Tabelas 3.1 e 3.2). Em 10 das 11 comunidades encontradas um animal vendido têm mais chance de permanecer em sua própria comunidade (comprado por um nó interno à comunidade) do que ir para outra comunidade (Tabela 3.1). Embora a comunidade 3 mostre uma fraca preferência em comercializar animais internamente, devido em grande parte ao seu intenso comércio com a comunidade 5 e 6 (Tabelas 3.1 e 3.2), o comércio interno é ainda maior do que o comércio desta com qualquer outra comunidade. 
Figura 3.3 - Circuitos pecuários do Mato Grosso.

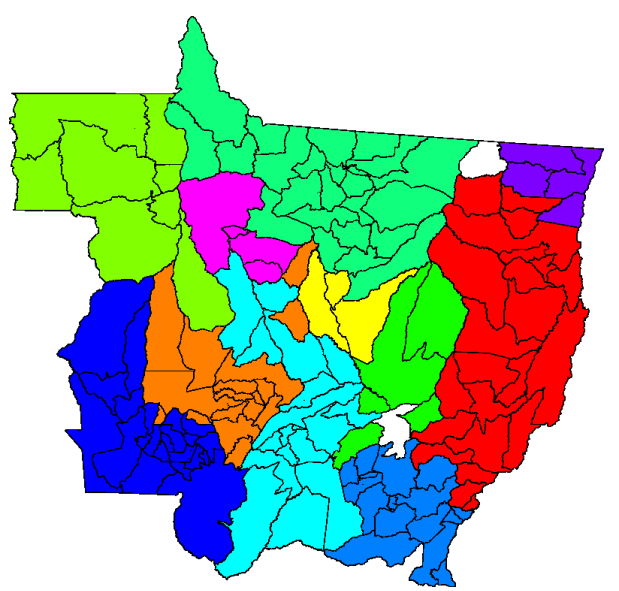

(a) Municípios

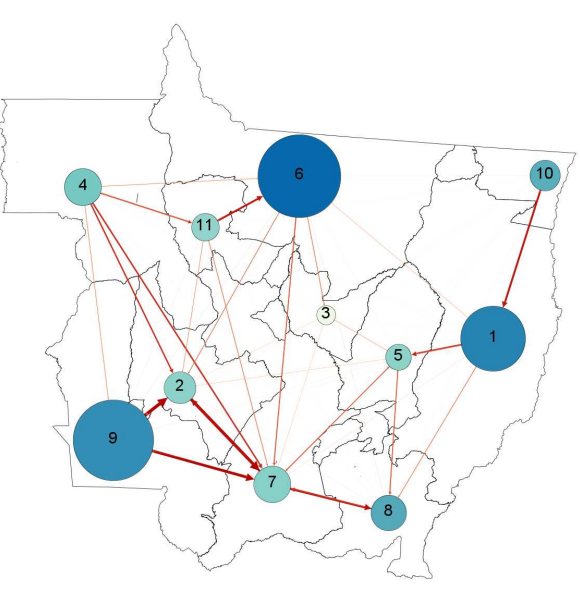

(b) Fluxo de animais

Figura 3.3 - Legenda:

A figura 3.3(a) mostra as 11 comunidades encontradas (municípios em branco permaneceram desagrupados). A figura 3.3(b) mostra o comércio entre comunidades: números representam a identificação de cada comunidade (correspondendo às Tabelas 3.1 e 3.2); a intensidade da cor e o tamanho das arestas estão correlacionados com o número de animais comercializados entre duas comunidades; o tamanho dos nós depende do número de animais transitados dentro de uma comunidade; e a intensidade de cor dos nós depende da probabilidade de que um animal vendido permaneça em sua própria comunidade (diagonal da Tabela 3.1). 
Tabela 3.1 - Movimentos de saída (\%). $A_{i j}=\frac{T_{i j}}{\sum i_{o u t}}$ onde $T_{i j}$ é o número de animais movimentados da comunidade $i$ para a comunidade $j$, e $\sum i_{\text {out }}$ é o número de animais vendidos pela comunidade $i$ (grau de saída, $K_{i \text { out }}$ ). Comunidades mostram clara preferência de vender animais internamente. O comércio realizado pelos 2 municípios não agrupados foi omitido.

\begin{tabular}{rrrrrrrrrrrrr}
\hline & 1 & 2 & 3 & 4 & 5 & 6 & 7 & 8 & 9 & 10 & 11 & Total de Animais \\
\hline 1 & 91,60 & 0,02 & 0,00 & 0,00 & 3,68 & 0,69 & 0,27 & 1,44 & 0,02 & 1,56 & 0,00 & 2.519 .752 \\
2 & 0,02 & 71,31 & 0,13 & 0,54 & 1,35 & 0,80 & 19,21 & 0,17 & 6,26 & 0,00 & 0,08 & 924.083 \\
3 & 0,00 & 10,54 & 39,23 & 0,00 & 23,73 & 10,69 & 14,72 & 0,04 & 1,05 & 0,00 & 0,00 & 89.107 \\
4 & 0,00 & 6,53 & 0,00 & 76,02 & 0,01 & 2,17 & 7,04 & 0,02 & 2,41 & 0,00 & 5,80 & 1.228 .075 \\
5 & 5,56 & 0,30 & 0,73 & 0,00 & 70,91 & 0,27 & 8,78 & 10,57 & 0,42 & 0,00 & 0,01 & 523.644 \\
6 & 0,08 & 1,11 & 1,07 & 0,19 & 0,00 & 94,70 & 1,74 & 0,18 & 0,14 & 0,00 & 0,74 & 3.371 .829 \\
7 & 0,28 & 9,44 & 0,14 & 0,17 & 3,23 & 1,11 & 72,46 & 9,73 & 2,77 & 0,00 & 0,37 & 1.265 .583 \\
8 & 2,14 & 0,42 & 0,00 & 0,02 & 3,39 & 0,20 & 8,17 & 83,66 & 0,78 & 0,01 & 0,01 & 1.016 .299 \\
9 & 0,02 & 5,31 & 0,01 & 0,22 & 0,02 & 0,07 & 4,50 & 0,16 & 89,60 & 0,01 & 0,07 & 3.439 .689 \\
10 & 15,18 & 0,00 & 0,02 & 0,00 & 0,22 & 0,33 & 0,00 & 0,03 & 0,01 & 82,57 & 0,01 & 724.604 \\
11 & 0,00 & 4,10 & 0,05 & 3,26 & 0,00 & 16,66 & 5,52 & 0,01 & 0,57 & 0,00 & 69,83 & 656.241 \\
\hline
\end{tabular}


Tabela 3.2 - Movimentos de entrada (\%). $A_{i j}=\frac{T_{i} j}{\sum_{i} j_{i n}}$ onde $T_{i j}$ é o número de animais movimentados da comunidade $i$ para a comunidade $j$, e $\sum j_{i n}$ é o número de animais comprados pela comunidade $j$ (grau de entrada, $K_{j}$ in). O comércio realizado pelos 2 municípios não agrupados foi omitido.

\begin{tabular}{rrrrrrrrrrrrr}
\hline & 1 & 2 & 3 & 4 & 5 & 6 & 7 & 8 & 9 & 10 & 11 \\
\hline 1 & 92,38 & 0,05 & 0,01 & 0,00 & 15,88 & 0,52 & 0,43 & 3,32 & 0,01 & 6,08 & 0,01 \\
2 & 0,01 & 58,75 & 1,51 & 0,51 & 2,13 & 0,22 & 11,19 & 0,14 & 1,79 & 0,00 & 0,12 \\
3 & 0,00 & 0,84 & 44,54 & 0,00 & 3,62 & 0,28 & 0,83 & 0,00 & 0,03 & 0,00 & 0,00 \\
4 & 0,00 & 7,15 & 0,03 & 95,60 & 0,02 & 0,78 & 5,45 & 0,02 & 0,92 & 0,00 & 12,67 \\
5 & 1,16 & 0,14 & 4,89 & 0,00 & 63,59 & 0,04 & 2,90 & 5,05 & 0,07 & 0,00 & 0,01 \\
6 & 0,10 & 3,34 & 45,79 & 0,67 & 0,03 & 94,19 & 3,71 & 0,54 & 0,14 & 0,00 & 4,41 \\
7 & 0,14 & 10,65 & 2,18 & 0,22 & 6,99 & 0,42 & 57,81 & 11,23 & 1,09 & 0,00 & 0,82 \\
8 & 0,87 & 0,38 & 0,04 & 0,02 & 5,90 & 0,06 & 5,23 & 77,57 & 0,25 & 0,01 & 0,02 \\
9 & 0,03 & 16,30 & 0,42 & 0,78 & 0,14 & 0,08 & 9,76 & 0,49 & 95,58 & 0,03 & 0,46 \\
10 & 4,40 & 0,00 & 0,15 & 0,00 & 0,27 & 0,07 & 0,00 & 0,02 & 0,00 & 92,58 & 0,01 \\
11 & 0,00 & 2,40 & 0,44 & 2,19 & 0,00 & 3,23 & 2,28 & 0,01 & 0,12 & 0,00 & 81,47 \\
Total de Animais & 2.498 .589 & 1.121 .681 & 78.501 & 976.503 & 583.919 & 3.390 .257 & 1.586 .209 & 1.096 .007 & 3.224 .366 & 646.296 & 562.458 \\
\hline
\end{tabular}




\section{DISCUSSÃO}

Embora a técnica de arrefecimento simulado tenha encontrado várias partições distintas, esses resultados mostram uma notória similaridade entre si, concordando na alocação em comunidades da maioria dos municípios. Redes que mostram mais de uma boa partição são comuns e esperadas. Isso apresenta um obstáculo para escolher, dentre várias partições, qual seria a correta. No entanto, é possível ver essa característica como uma vantagem, dado que possibilita a escolha da partição que melhor atenda às necessidades de um pesquisador ou tomador de decisão. Extrair as concordâncias entre as diferentes partições é uma maneira útil de obter grupos pequenos que podem ser posteriormente aglomerados de maneira a atender os objetivos específicos de um estudo sem violar a definição de comunidade escolhida. Quando aplicada à uma rede de trânsito animal utilizando a definição proposta por KIM, SON e JEONG (2010), essa solução final é consistente com a idéia de circuitos pecuários, mostrando que um animal vai mais provavelmente permanecer em sua comunidade ao invés de ser vendido para (ou ser comprado de) outra comunidade qualquer.

A solução final apresenta um padrão geográfico e comercial claro, característica crucial para aplicações em medicina veterinária preventiva, além de possuir uma interpretação clara e útil em redes de comércio onde ligações emergem das escolhas de nós comerciantes, e esses últimos são fortemente influenciados pelos "hubs" (nós com alto valor de PageRank), os quais normalmente apresentam alta competitividade quando comparados com outros nós da rede. 


\section{CONCLUSÕES}

Vimos que o método descrito nessa tese pode revelar a estrutura comunitária de uma rede direcionada de trânsito animal. Acreditamos que essa abordagem é apropriada para redes de trânsito animal e circuitos pecuários, assim como outras redes onde o caminho percorrido por um passeador aleatório (ou as escolhas do nós em estabelecer ligações com outros noś) está incluída na definição de comunidade. Essa abordagem pode ser utilizada para melhor compreender o fluxo interno de uma rede, ajudar no planejamento de um sistema de vigilância baseado em risco, melhorar modelos preditivos, guiar uma amostra estratificada, determinar áreas alvos para programas sanitários e definir zonas para restrição de movimentação. 


\section{REFERÊNCIAS}

BASTIAN, M.; HEYMANN, S.; JACOMY, M. Gephi: An Open Source Software for Exploring and Manipulating Networks. In: INTERNATIONAL AAAI CONFERENCE ON WEBLOGS AND SOCIAL MEDIA. San Jose, California, 2009.

BIGRAS-POUlin, M.; THOMPSON, R. A.; CHRIEL, M.; MORTENSEN, S.; GREINER, M. Network analysis of Danish cattle industry trade patterns as an evaluation of risk potential for disease spread. Preventive Veterinary Medicine, vol. 76, no. 1-2, pp. 11-39, 2006. doi:10.1016/j.prevetmed.2006.04.004.

BIVAND, R. S.; PEBESMA, E. J.; GOMEZ-RUBIO, V. Applied spatial data analysis with R. New York, New York, USA, 2008.

COX, T.; COX, M. Multidimensional Scaling, Second Edition, Monographs on Statistics 85 Applied Probability, vol. 88. Chapman and Hall, 2000. doi:10.1201/9781420036121.

FORTUNATO, S. Community detection in graphs. Physics Reports, vol. 486, no. 3-5, pp. $75-174,2010$.

FRITSCH, A. MCCLUST: process an MCMC sample of clusterings, 2009. URL http: //cran.r-project.org/package=mcclust.

GIBBEnS, J. C.; WILESMith, J. W.; SHARPE, C. E. Descriptive epidemiology of the 2001 foot-and-mouth disease epidemic in Great Britain: the first five months.

Veterinary Record, vol. 149, no. 24, p. 729-743, 2001.

GOOD, B. H.; DE MONTJOYE, Y.-A.; CLAUSET, A. Performance of modularity maximization in practical contexts. Physical Review E, vol. 81, no. 4, p. 20, 2010. doi:10.1103/PhysRevE.81.046106.

GREEN, D. M.; GREGORY, A.; MUNRO, L. A. Small- and large-scale network structure of live fish movements in Scotland. Preventive Veterinary Medicine, vol. 91, no. 2-4, pp. 261-9, 2009.

GREEN, D. M.; WERKMAN, M.; MUNRO, L. A.; KAO, R. R.; KISS, I. Z.; DANON, L. Tools to study trends in community structure: application to fish and livestock trading networks. Preventive Veterinary Medicine, vol. 99, no. 2-4, pp. 225-8, 2011.

GUIMERÀ, R.; AMARAL, L. N. Cartography of complex networks: modules and universal roles. Journal of Statistical Mechanics (Online), vol. 2005, no. P02001, p. nihpa35573, 2005. doi:10.1088/1742-5468/2005/02/P02001.

HARVEY, N.; REEVES, A.; SCHOENBAUM, M. A.; ZAGMUTT-VERGARA, F. J.; DUBÉ, C.; HILL, A. E.; CORSO, B. A.; MCNAB, W. B.; CARTWRIGHT, C. I.; SALMAN, M. D. The North American Animal Disease Spread Model: a simulation model to assist decision making in evaluating animal disease incursions. Preventive Veterinary Medicine, vol. 82, no. 3-4, pp. 176-97, 2007.

INSTITUTO BRASILEIRO DE GEOGRAFIA E ESTATÍSTICA. Pesquisa Pecuária Municipal, 2010. URL

http://www . sidra. ibge.gov . br/bda/tabela/listabl . asp?c=73\&z=p\&o=29. 
KIM, Y.; SON, S.-W.; JEONG, H. Finding communities in directed networks. Physical Review E, vol. 81, no. 1, pp. 1-9, 2010. doi:10.1103/PhysRevE.81.016103.

KIRKPATRICK, S.; GELATT, C. D. J.; VECCHI, M. P. Optimization by Simulated Annealing. Science, vol. 220, no. 4598, pp. 671-680, 1983.

doi:10.1126/science.220.4598.671.

LANGVILLE, A. N.; MEYER, C. D. Google's PageRank and beyond: the science of search engine rankings. Princeton University Press, Princeton, New Jersey, 2006.

LEICHT, E.; NEWMAN, M. E. J. Community Structure in Directed Networks.

Physical Review Letters, vol. 100, no. 11, pp. 1-4, 2008.

doi:10.1103/PhysRevLett.100.118703.

LENTZ, H. H. K.; KONSCHAKE, M.; TESKE, K.; KASPER, M.; ROTHER, B.; CARMANNS, R.; PETERSEN, B.; CONRATHS, F. J.; SELHORST, T. Trade communities and their spatial patterns in the German pork production network. Preventive Veterinary Medicine, vol. 98, no. 2-3, pp. 176-81, 2011.

LEWIN-KOH, N. J.; BIVAND, R.; PEBESMA, E. J.; ARCHER, E.; BADDELEY, A.; BIBIKO, H.-J.; DRAY, S.; FORREST, D.; FRIENDLY, M.; GIRAUDOUX, P.; GOLICHER, D.; RUBIO, V. G.; HAUSMANN, P.; HUFTHAMMER, K. O.; JAGGER, T.; LUQUE, S. P.; MACQUEEN, D.; NICCOLAI, A.; SHORT, T.; STABLER, B.; TURNER, R. Maptools: tools for reading and handling spatial objects, 2011. URL http://cran.r-project.org/package=maptools.

LUSSEAU, D.; NEWMAN, M. E. J. Identifying the role that animals play in their social networks. Proceedings. Biological sciences / The Royal Society, vol. 271, pp. 5477-5481, 2004.

LUSSEAU, D.; SCHNEIDER, K.; BOISSEAU, O. J.; HAASE, P.; SLOOTEN, E.; DAWSON, S. M. The bottlenose dolphin community of Doubtful Sound features a large proportion of long-lasting associations. Behavioral Ecology and Sociobiology, vol. 54, no. 4, pp. 396-405, 2003. doi:10.1007/s00265-003-0651-y.

MARTÍNEZ-LÓPEZ, B.; PEREZ, A. M.; SÁNCHEZ-VIZCAÍNO, J. M. Social network analysis. Review of general concepts and use in Preventive Veterinary Medicine.

Transboundary and Emerging Diseases, vol. 56, no. 4, pp. 109-20, 2009. doi:10.1111/j.1865-1682.2009.01073.x.

MEILA, M. Comparing clusterings - an information based distance. Journal of Multivariate Analysis, vol. 98, no. 5, pp. 873-895, 2007.

doi:10.1016/j.jmva.2006.11.013.

NEWMAN, M.; GIRVAN, M. Finding and evaluating community structure in networks. Physical Review E, vol. 69, no. 2, pp. 1-15, 2004. doi:10.1103/PhysRevE.69.026113.

PAGE, L.; BRIN, S.; MOTWANI, R.; WINOGRAD, T. The PageRank citation ranking: bringing order to the web. Stanford InfoLab, 1998.

R DEVELOPMENT CORE TEAM. R: A language and environment for statistical computing., 2010. doi:ISBN3-900051-07-0. URL http://www.r-project.org. 
R DEVELOPMENT CORE TEAM. Foreign: read data stored by minitab, S, SAS, SPSS, Stata, Systat, dBase, ..., 2011. URL

http://cran.r-project.org/package=foreign.

SALATHÉ, M.; JONES, J. H. Dynamics and control of diseases in networks with community structure. PLoS Computational Biology, vol. 6, no. 4, p. e1000736, 2010. doi:10.1371/journal.pcbi.1000736.

SCHNUTE, J. T.; BOERS, N.; HAIGH, R.; COUTURE-Beil, A. PBSmapping: mapping fisheries data and spatial analysis tools, 2010. URL

http://cran.r-project.org/package=PBSmapping.

WORLD ORGANISATION FOR ANIMAL HEALTH. Terrestrial Animal Health Code, vol. 33. World Organisation for Animal Health, San Francisco, California, 2010. URL www. oie.int. 